\title{
Detection of Gluten Exorphin B4 and B5 in Human Blood by Liquid Chromatography-Mass Spectrometry/Mass Spectrometry
}

\author{
Christopher L. Pennington ${ }^{1}$, Craig P. Dufresne ${ }^{2}$, Giuseppe Fanciulli ${ }^{3}$ and Troy D. Wood*,1,4 \\ ${ }^{I}$ Department of Chemistry, University at Buffalo, State University of New York, Buffalo, NY, USA \\ ${ }^{2}$ ThermoFisher Scientific, West Palm Beach, FL, USA \\ ${ }^{3}$ Dipartimento-Struttura Clinica Medica-Patologia Speciale Medica, University of Sassari, Sassari, Italy \\ ${ }^{4}$ Also Department of Structural Biology, University at Buffalo, State University of New York, Buffalo, NY, USA
}

\begin{abstract}
Liquid chromatography-tandem mass spectrometry was employed to monitor for gluten exorphins (GEs) in blood from samples collected from Celiac Disease subjects after the consumption of a physiological meal. GE-B4 and B5 were detected in three of the four subjects, the first time GEs have been detected in human blood.
\end{abstract}

\section{INTRODUCTION}

Literature reports suggest that the digestion of food proteins may produce peptides having opiate effects; these peptides are called "exorphins" because of their exogenous (alimentary) origin and opioid-like activity [1]. Gluten exorphins (GEs) are a family of five opioid-peptides designated GE-A4, Gly-Tyr-Tyr-Pro; GE-A5, Gly-Tyr-Tyr-Pro-Thr; GE-B4, Tyr-Gly-Gly-Trp; GE-B5, Tyr-Gly-Gly-Trp-Leu; and GE-C, Tyr-Pro-Ile-Ser-Leu, that were originally identified, in vitro, from the enzymatic digestion of high molecular wheat gluten [2-4]. It is proposed that GEs enter the blood stream by passing through intestinal lining that is compromised by acquired or genetic conditions, reach the central nervous system where they modulate neurotransmission and affect behavior ("opioid excess theory", OET), possibly playing a pathogenetic role in some mental disturbances [5].

A key aspect of the OET is the ability of GEs, once produced in the gastrointestinal tract, to cross the intestinal barrier and enter the blood stream. To date, however, the detection of GEs in human blood has not been reported. Here an ultrafiltration method followed by electrospray ionization (ESI) liquid chromatography-tandem mass spectrometry (LC-MS/MS) is used to determine if GEs can be detected in blood samples from subjects with compromised intestinal lining. Subjects with gluten-induced enteropathy (Celiac Disease, CD) were chosen as model subjects, because CD is well-known to result in increased intestinal permeability to macromolecules [6-8], and is frequently associated with mental disorders [9-11].

$\mathrm{CD}$ is a disorder characterized by an immunologically mediated enteropathy induced in genetically susceptible individuals by gluten ingestion [12]. Although most of the complications of CD (chronic diarrhea, anemia, osteoporosis, failure to thrive, etc.) are due to the reduction of intestinal absorption of nutrients from food, CD is associated with conditions such as mental disorders [9-11] that are unlikely to be due to malabsorption of nutrients. Interestingly, the

*Address correspondence to this author at the Department of Chemistry, University at Buffalo, State University of New York, Buffalo, NY, USA; Email: twood@buffalo.edu gluten-induced enteropathy not only reduces the body's ability to adsorb nutrients, but also increases the intestinal permeability to macromolecules [6-8].

It has been hypothesized that increased intestinal permeability might facilitate the absorption of dietary opioid peptides able to affect behavior [13]. In animal models, GE-B5 has been shown to stimulate the glucose-induced insulin release after intragastric administration [14]. It was previously demonstrated that GE-B5 can play a regulatory role on pituitary secretion, since it markedly stimulates Prolactin secretion in rats $[15,16]$, probably via a reduction in neurotransmitter release at the hypothalamic level.

No effects of GE-B4 determinations are reported in the literature. GE-B4, in particular, was unable to stimulate Prolactin secretion in animal models, in contrast to GE-B5 [17].

In this study, experimental subjects were given a "pizza meal" (a meal widely used as a standard meal in experiments in humans [18-20], to simulate "one of the most common postprandial physiologic conditions in modern society") [20], in the hypothesis that the likelihood of finding GEs in blood was higher after gluten ingestion. Compared to high abundance plasma proteins [21], GEs, with molecular weights ranging from $481 \mathrm{Da}$ for GE-B4 to $594 \mathrm{Da}$ for GEB5, are relatively small. Given the complexity of plasma samples, and the anticipated low abundance of GEs in plasma, the use of sample preparation methodology that allowed for the isolation and enrichment of low molecular weight plasma peptides was essential. In addition, because opioid peptides can be degraded by plasmatic proteases [2224], the use of a protease inhibitor is imperative to ensure that peptide degradation does not continue after blood samples are obtained [25]. Enrichment and detection of GEs was accomplished by adapting existing ultrafiltration sample preparation methods [26, 27], and then subjecting the low molecular weight plasma fraction to LC-MS/MS analysis. The protease inhibitor used to prevent degradation of the target peptides was aprotinin, which has been shown to prevent GE-A5 degradation in biological fluids [28]. Thus, the use of this sample preparation approach may facilitate the 
observation of the two GE peptides, GE-B4 and B5, in plasma samples obtained from patients with $\mathrm{CD}$.

\section{METHODS}

\section{Materials}

Aprotinin was purchased from Boehringer-Mannheim (Mannheim, Germany). Standard GE peptides GE-A4, GEA5, GE-B4, and GE-B5 were synthesized in house as described elsewhere [14] (GE-C was not available). The $4 \mathrm{~mL}$ glass centrifuge tubes used for sample collection, containing $0.048 \mathrm{~mL}$ of a $15 \%$ potassium ethylenediaminetetraacetic acid solution as an anticoagulant, were from BD Vacutainer (Plymouth, UK). Sodium azide $\left(\mathrm{NaN}_{3}\right)$ was from Sigma (Milan, Italy). Centricon centrifugal filters containing a YMmembrane with a $3000 \mathrm{Da}$ nominal molecular weight cutoff (NMWCO) were purchased from Millipore (Billerica, MA). Acetonitrile (ACN) and water were from J.T. Baker (Philipsburg, NJ). High purity formic acid was from Sigma Aldrich (St. Louis, MO) and ammonium bicarbonate was from Fisher Scientific (Pittsburgh, PA).

\section{Subjects and Sample Collection}

All subjects (or their parents if under the age of 18) gave informed consent to enter the study, which was approved by the Ethical Committee of the University of Sassari and the Institutional Review Board of the University at Buffalo. The subjects consisted of three females (subject I, age 40; subject II, age 33; subject III, age 33) and one male (subject IV, age 15) all of whom were from Sardinia, Italy. At the time of the blood collection all subjects were suspected of being affected by CD based on serological tests [29], but were not yet participating in a gluten free diet. Diagnosis of CD was confirmed in all patients by duodenal biopsy that was performed within one week of the sample collection.

On the day of sample collection, all subjects consumed a "pizza meal" that consisted of a cheese pizza (250 - 270 grams) made with wheat based dough, tomato sauce, and oil. Blood samples ( $2 \mathrm{~mL} /$ time point) were collected, through an intravenous catheter inserted into the forearm, at $\mathrm{h} 01.00 \mathrm{PM}$ (time point 0 , immediately before the "pizza meal" started), and then after at 15, 30, 45, 60, 75, 90, and 120 min. All subjects finished consumption of their pizza between the collection of the 30 and 45 min time point samples. Subjects participating in the study were not given specific instructions to fast prior to participating in the study. During the sample collection all subjects were permitted to drink water, sit in a chair, or walk around.

Blood samples were collected into the glass centrifuge tubes. Immediately upon collection, samples were stored on ice in a cooler until centrifugation at 5,000g for $10 \mathrm{~min}$. Following centrifugation, $500 \mu \mathrm{L}$ of each plasma sample was transferred into a separate polypropylene tube and treated with a $10 \%$ solution of $\mathrm{NaN}_{3}$ as an antibacterial preservative, at ratio of $10 \mu \mathrm{L} 10 \% \mathrm{NaN}_{3} / \mathrm{mL}$ plasma. Plasma samples were also treated with a protease inhibitor (aprotinin) at a ratio of $2.5 \mu \mathrm{g}$ aprotinin $(10 \mu \mathrm{L}) / \mathrm{mL}$ of plasma. While protocols from the Human Proteome Organization (HUPO) often call for using a protease inhibitor cocktail, individual protease inhibitors such as aprotinin have shown virtually equivalent inhibitory action [25]. Following centrifugation and treatment the plasma samples were stored in a freezer at -80
${ }^{\circ} \mathrm{C}$ until shipment on dry ice via courier service to the University at Buffalo (Buffalo, NY).

\section{LC-MS/MS Analysis}

Upon arrival at The University at Buffalo, plasma samples were stored at $-80{ }^{\circ} \mathrm{C}$. For each patient, time points at 0 , $15,30,45,60,75,90$, and 120 min were analyzed. On the day of analysis, plasma samples were removed from the cryofreezer and thawed at room temperature. For each time point, the entire plasma sample was diluted with $3 \mathrm{~mL}$ of 20 $\mathrm{mM}$ ammonium bicarbonate $(\mathrm{pH}=8)$ and $1 \mathrm{~mL}$ of ACN. The final volume of all samples was adjusted to $5 \mathrm{~mL}$ with $20 \mathrm{mM}$ ammonium bicarbonate as needed. Samples were shaken by hand, vortexed, and allowed to stand a room temperature for $20 \mathrm{~min}$. After $20 \mathrm{~min}$ samples were shaken by hand, vortexed a second time, and divided equally among four $1.5 \mathrm{~mL}$ microcentrifuge tubes and centrifuged at 20,000 $g$ and $4{ }^{\circ} \mathrm{C}$ for $90 \mathrm{~min}$, using a Sorvall Legend RT centrifuge (Thermo Fisher Scientific, Asheville, NC). This procedure was performed in order to remove any particulates that might otherwise clog the $3000 \mathrm{Da}$ NMWCO filter's membrane.

Prior to use, the 3000 NMWCO filters were rinsed according to the manufacturer's instructions with $2 \mathrm{~mL}$ of 20 $\mathrm{mM}$ ammonium bicarbonate. Following filter pre-rinsing, the supernatant from the four microcentrifuge tubes was split evenly among two 3000 NMWCO filter units. Ultrafiltration was carried out similar to previous methods [26, 27] but by centrifuging the $3000 \mathrm{NMWCO}$ filter units at $3000 \mathrm{~g}$ overnight $(\sim 12-15 \mathrm{hrs})$ at $4{ }^{\circ} \mathrm{C}$, until at least $80 \%$ of the liquid passed through the membrane. The extended centrifugation time was due to the use of a swing bucket rotor instead of a recommended fixed angle rotor. Following centrifugation, the low molecular weight fractions for each sample were pooled, frozen on dry ice, and lyophilized using a Labconco Free Zone 2.3 freeze dryer (Kansas City, MO). After freeze drying, samples were returned to the cryofreezer until shipment to the Thermo Fisher Scientific Training Institute (West Palm Beach, FL) for LC-MS/MS analysis.

LC-MS/MS analysis was conducted using a Surveyor HPLC equipped with a Micro AS auto sampler interfaced, through an Ion Max ESI source, to a LTQ-Orbitrap ${ }^{\mathrm{TM}}$ hybrid mass spectrometer (Thermo Electron, Bremen, Germany) [30-32]. Separations were carried out on a Thermo Fisher GOLD (100 x $2.1 \mathrm{~mm}$ ID) $\mathrm{C}_{18}$ column with $1.9 \mu \mathrm{m}$ packing material, and operated at a $200 \mu \mathrm{L} / \mathrm{min}$ flow rate with mobile phases $\mathrm{A}: \mathrm{H}_{2} \mathrm{O}+0.1 \%$ formic acid and $\mathrm{B}$ : $\mathrm{ACN}+0.1 \%$ formic acid. The LC gradient ran from $2 \% \mathrm{~B}$ at $0 \mathrm{~min}$ to $60 \%$ $\mathrm{B}$ at $37 \mathrm{~min}$, where it was held for $2 \mathrm{~min}$, until being increased to $98 \%$ B over the next $5 \mathrm{~min}$. Following the run, the column was equilibrated at initial conditions for $15 \mathrm{~min}$ prior to the next injection. The injection volume was $6 \mu \mathrm{L}$ and all samples were redissolved in $100 \mu \mathrm{L}$ of mobile phase A. Prior to analysis the column was equilibrated at initial conditions for $15 \mathrm{~min}$ prior to the next injection. The injection volume was $6 \mu \mathrm{L}$ and all samples were redissolved in $100 \mu \mathrm{L}$ of mobile phase A prior to analysis.

MS analysis on the LTQ-Orbitrap used the $\mathrm{LTQ}^{\mathrm{TM}}$ linear ion trap to specifically acquire MS/MS spectra of target GEs peptides A4, A5, B4, B5, and C, while the Orbitrap FTMS was used to acquire a high resolution $(\mathrm{R}=30,000)$ full MS scan of all ions from m/z 400 to m/z $2000(\mathrm{R}=7,500 @ \mathrm{~m} / z$ 


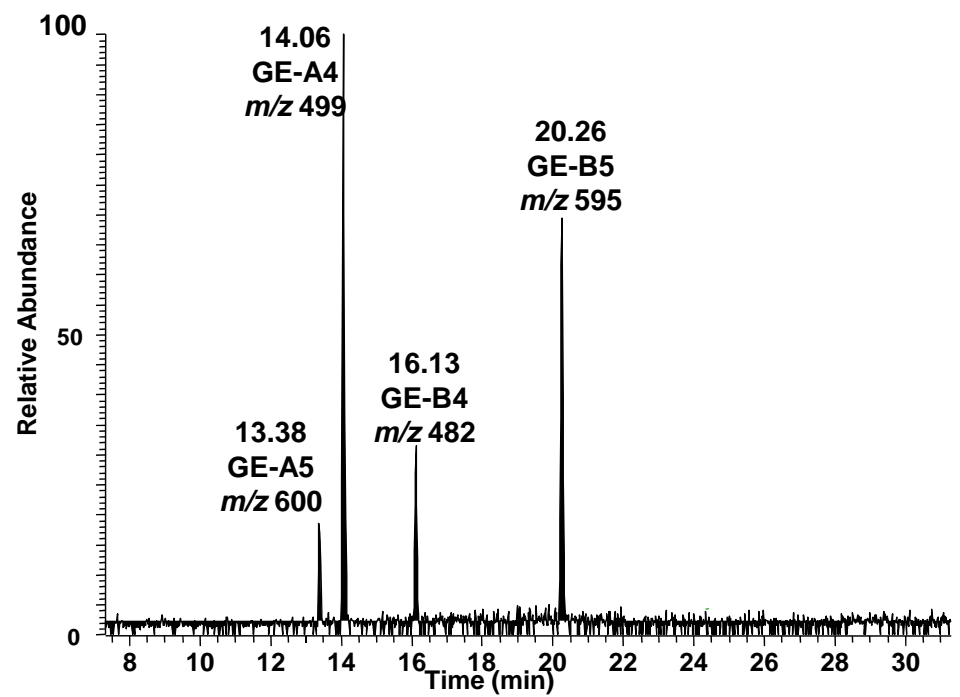

Fig. (1). Total ion chromatogram showing retention times of GE standards in order of elution: A5 (13.38 min), A4 (14.06 min), B4 (16.13 $\mathrm{min}$ ), and B5 (20.26 min).

400 is the lowest resolution possible). Theoretical masses for all GE peptides were calculated using Xcalibur's QualBrowser program (ThermoFisher). A $2 \mu \mathrm{M}$ GE test mixture comprised of GE-A4, A5, B4, and B5 was analyzed to establish retention times for the GEs and provided MS/MS reference mass spectra. Since GE-C was not available as a standard to provide an expected retention time, targeted MS/MS of its $(\mathrm{M}+\mathrm{H})^{+}$peak at $\mathrm{m} / z 592$ was performed concurrent with the other GE.

\section{RESULTS AND DISCUSSION}

A total ion chromatogram indicating the order of elution for the four GE standard peptides is displayed in (Fig. 1). MS/MS spectra for each of the GE standards is provided in (Fig. 2). Identification of GEs in the plasma samples was based on a retention time match with the standard mixture, observation of characteristic diagnostic ions in the MS/MS spectra, and, when available, a high resolution spectrum obtained for the $(\mathrm{M}+\mathrm{H})^{+}$ion in the Orbitrap full MS scan. Util-

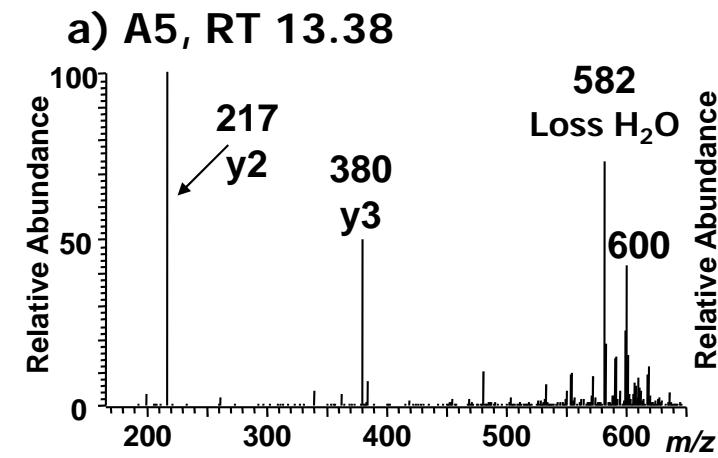

b) A4, RT 14.06
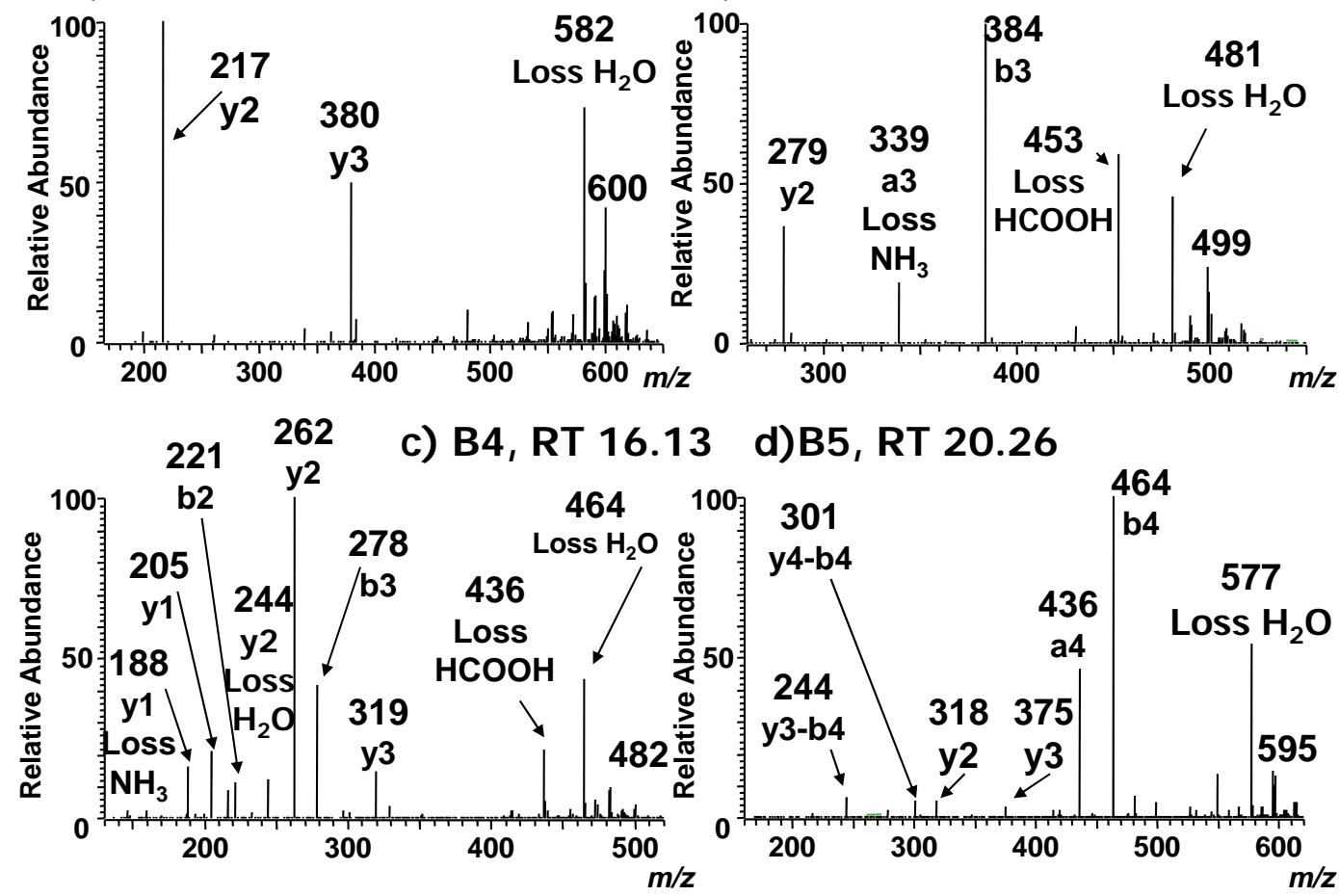

Fig. (2). Tandem mass spectra of standard GEs: a) A5, b) A4, c) B4, d) B5. 
Table 1. Summary of Samples in which GEs were Identified

\begin{tabular}{|c|c|c|c|c|c|c|c|c|c|}
\hline Std. & B4 & 16.13 & $1.98 \mathrm{E}+04$ & 4.30 & --- & $7 / 7(100 \%)$ & 482.2028 & -1.266 & --- \\
\hline Std. & B5 & 20.26 & $6.10 \mathrm{E}+04$ & 4.79 & --- & $6 / 6(100 \%)$ & 595.2874 & -0.125 & --- \\
\hline I-60 & B5 & 20.34 & $6.28 \mathrm{E}+02$ & 2.80 & 0.08 & $4 / 6(67 \%)$ & 595.2877 & 0.412 & 3 \\
\hline I-90 & B5 & 20.30 & $3.48 \mathrm{E}+02$ & 2.54 & 0.04 & $6 / 6(100 \%)$ & --- & --- & 3 \\
\hline I-120 & B5 & 20.29 & $3.70 \mathrm{E}+02$ & 2.57 & 0.03 & $4 / 6(67 \%)$ & --- & --- & 3 \\
\hline II-0 & B4 & 16.05 & $2.42 \mathrm{E}+02$ & 2.38 & -0.08 & $6 / 7(86 \%)$ & --- & --- & 3 \\
\hline II-0 & B5 & 21.21 & $3.55 \mathrm{E}+02$ & 2.55 & 0.95 & $5 / 6(83 \%)$ & --- & --- & 3 \\
\hline II-30 & B5 & 20.30 & $4.57 \mathrm{E}+02$ & 2.66 & 0.04 & $5 / 6(83 \%)$ & --- & --- & 3 \\
\hline III-90 & B4 & 16.09 & $3.09 \mathrm{E}+02$ & 2.49 & -0.04 & $6 / 7(86 \%)$ & --- & --- & 2 \\
\hline
\end{tabular}

Note: A total of six characteristic MS/MS fragment ions were used to identify Ge-B5. The characteristic fragment ions for GE-B5 were the b4 ( $/ 2 / z 464)$, a $4(\mathrm{~m} / z$ 436), y3 $(\mathrm{m} / z 375)$, y2 $(\mathrm{m} / \mathrm{z}, 318)$ and the y4-b4 $(\mathrm{m} / \mathrm{z} 301)$ and y3-b4 $(\mathrm{m} / \mathrm{z} 244)$ internal fragment ions. A total of seven characteristic MS/MS fragment ions were used to identify GE-B4. The characteristic fragment ions for GE-B4 were the y3 $(\mathrm{m} / \mathrm{z} 319)$, b3 $(\mathrm{m} / \mathrm{z} 278)$, y2 $(\mathrm{m} / \mathrm{z} 262)$, y2 loss of $\mathrm{H} 2 \mathrm{O}(\mathrm{m} / \mathrm{z} 244), \mathrm{y} 1(\mathrm{~m} / \mathrm{z} 205)$, and y1 loss of NH3 ion.

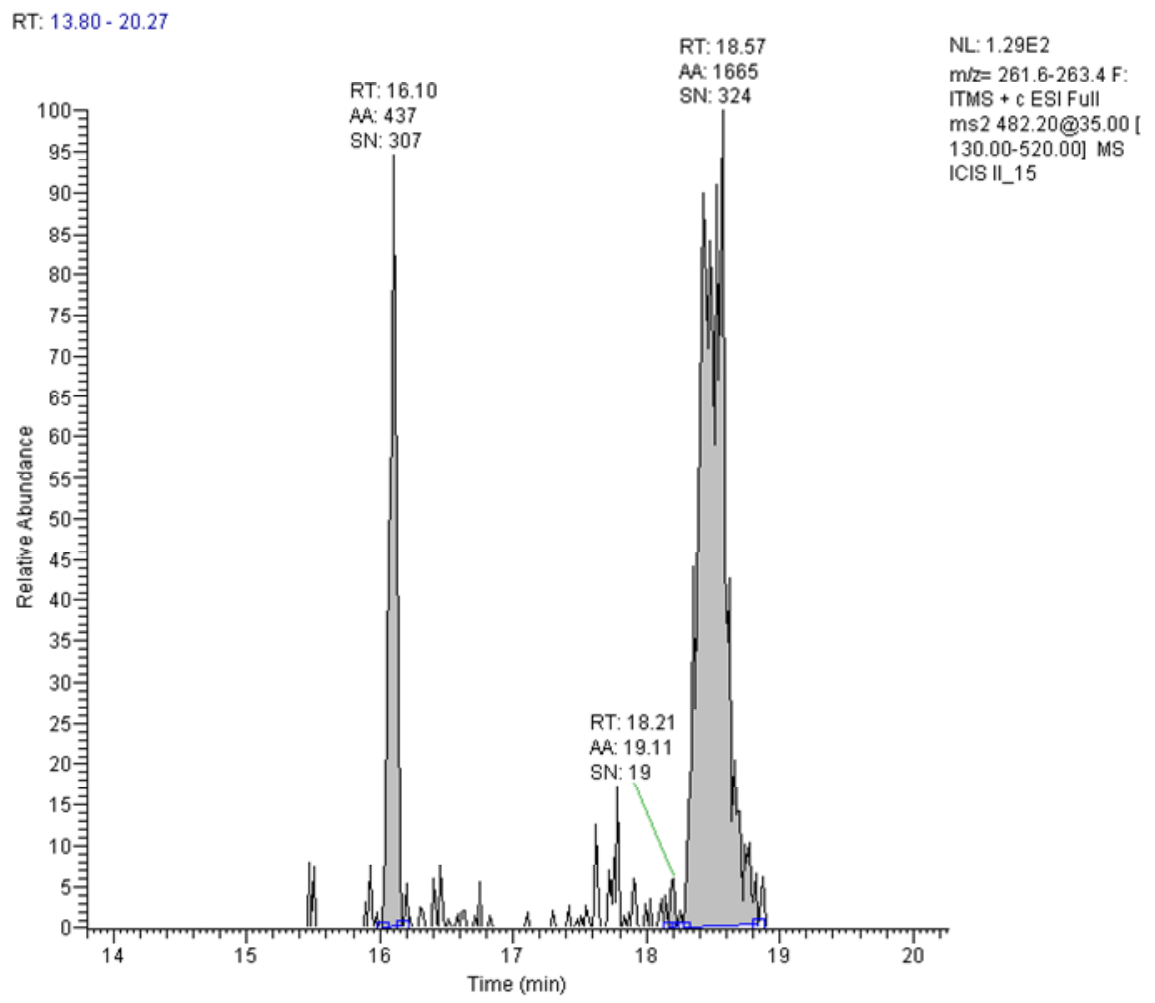

Fig. (3). Extracted ion chromatogram from sample II-15 produced by plotting the ion transition $\mathrm{m} / z$. $482 \rightarrow \mathrm{m} / z$. 262, with a peak at 16.01 minutes.

izing the described ultrafiltration LC-MS/MS methodology, GE-B4, $(\mathrm{M}+\mathrm{H})^{+}=482$, and GE-B5, $(\mathrm{M}+\mathrm{H})^{+}=595$, were identified in plasma samples from subjects I, II and III. These results are summarized in Tables 1 (GE-B4) and 2 (GE-B5).
A representative extracted ion chromatogram from sample II-15 (Fig. 3) shows a peak eluting at $16.01 \mathrm{~min}$, which matches the retention time of the GE-B4 standard. The chromatogram in (Fig. 3) is based on an ion transition $(\mathrm{m} / z$ $482 \rightarrow \mathrm{m} / z$ 262) that is indicative of GE-B4. The identifica- 
tion of GE-B4 is enhanced by the observation of several fragment ions characteristic of GE-B4, in the MS/MS spectrum of $\mathrm{m} / z 482$ (Fig. 4). GE-B4's identification in sample II-15 was further supported by the observation of a peak at $\mathrm{m} / z$ 482.2032 from the high resolution full MS Orbitrap scan. This peak matches the chemical formula for the GE-B4 $(\mathrm{M}+\mathrm{H})^{+}$ion $\left(\mathrm{C}_{24} \mathrm{H}_{28} \mathrm{O}_{6} \mathrm{~N}_{5}\right)$ within $0.5 \mathrm{ppm}$ of the theoretical mass. GE-B4 was also identified in the zero and $30 \mathrm{~min}$ time points from subject II as well as the 90 min time point from subjects I and III.

In all cases, the retention time differences between GEB4 observed in the plasma samples and the GE-B4 standard are within $10 \mathrm{~s}$. A total of seven characteristic MS/MS fragment ions were used to identify GE-B4. The characteristic fragment ions for GE-B4 were the y3 $(\mathrm{m} / \mathrm{z}, 319), \mathrm{b} 3(\mathrm{~m} / \mathrm{z}$ 278), y2 (m/z 262), y2 loss of $\mathrm{H} 2 \mathrm{O}(\mathrm{m} / \mathrm{z} 244)$, y1 $(\mathrm{m} / \mathrm{z} 205)$, and y1 loss of NH3 ion.

The characteristic GE-B5 ion transition $\mathrm{m} / z 595 \rightarrow \mathrm{m} / z$ 464 was used to plot an extracted ion chromatogram from sample I-60 (Fig. 5). The chromatogram in (Fig. 4) shows a peak at 20.34 min that matches the retention time of the GEB5 standard. The identity of GE-B5 was confirmed by the presence of characteristic fragment ions in the MS/MS spectra of $\mathrm{m} / z 595$ (Fig. 6). A total of six characteristic MS/MS fragment ions were used to identify GE-B5. The characteristic fragment ions for GE-B5 were the b4 $(\mathrm{m} / \mathrm{z} 464)$, a $4(\mathrm{~m} / \mathrm{z}$ 436), y3 $(\mathrm{m} / \mathrm{z}, 375), \mathrm{y} 2(\mathrm{~m} / \mathrm{z}, 318)$ and the y4-b4 $(\mathrm{m} / \mathrm{z} 301)$ and y3-b4 $(\mathrm{m} / \mathrm{z} 244)$ internal fragment ions.

However, the additional C-terminal leucine residue of GE-B5 causes unique y and internal fragment ions at $\mathrm{m} / z$ 375 (y3), 318 (y2), 301 (y4-b4), and 244 (y3-b4) to be observed. This, along with the matching retention time, allows

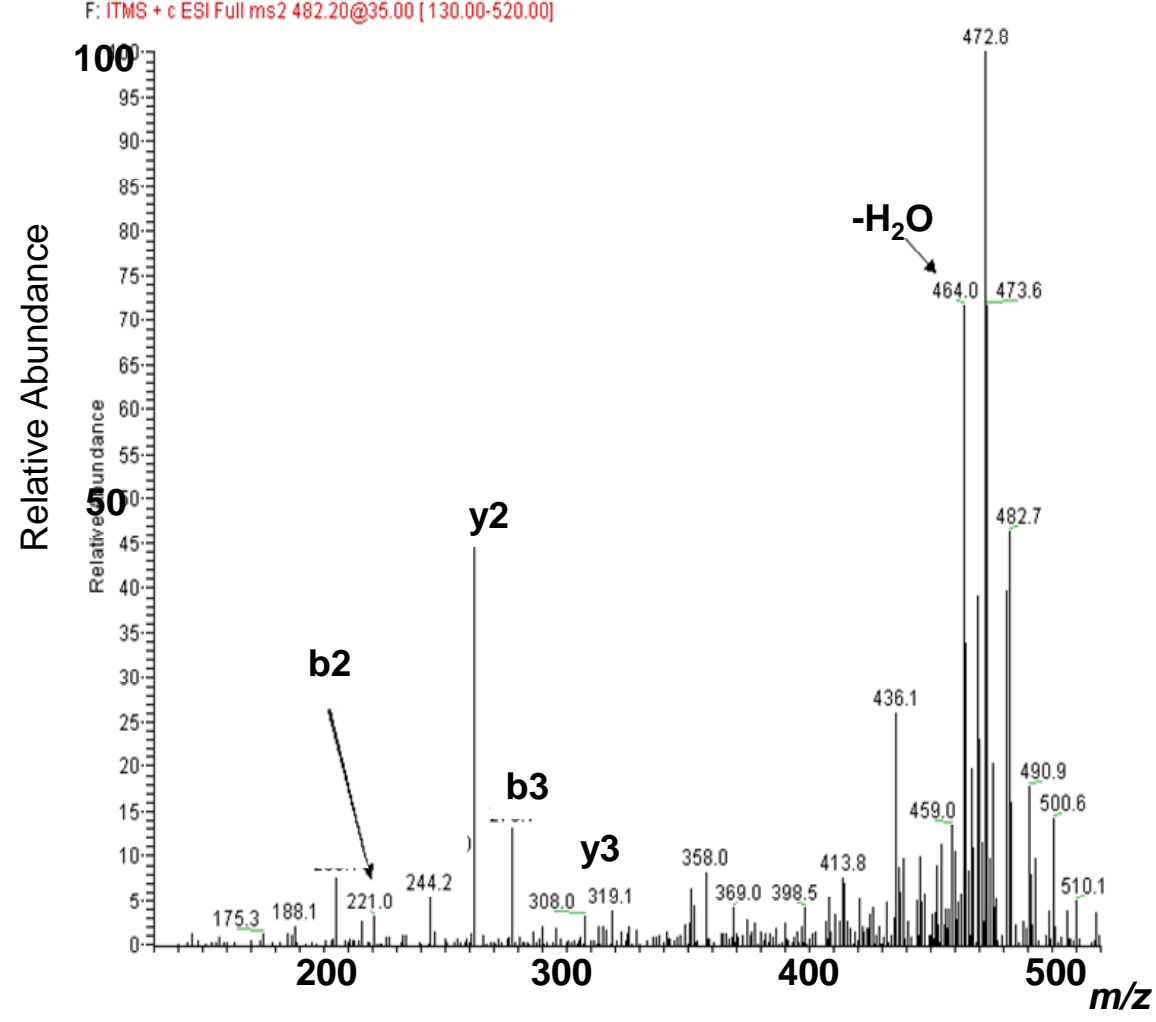

Fig. (4). MS/MS spectrum of m/z 482 from the chromatographic peak at 16.01 minutes from sample II-15. the unambiguous identification of GE-B5 in samples I-60, I75, I-90, I-120, II-0, II-15, and II-30. The identification of GE-B5 in sample I-60 was further bolstered by a small, but discernable, peak at $\mathrm{m} / z, 595.2877$ obtained in the high resolution full MS Orbitrap scan. This peak matches the chemical formula for the GE-B5 $(\mathrm{M}+\mathrm{H})^{+}$ion $\left(\mathrm{C}_{30} \mathrm{H}_{39} \mathrm{O}_{7} \mathrm{~N}_{6}\right)$ within $0.4 \mathrm{ppm}$ of the theoretical mass. Due to the observation of GE-B4 and B5 in the zero time point of subject II, a thorough re-inspection of all blank samples was conducted to check for the possibility of instrument carry over, which would provide a false positive result. Analysis of blank samples, including the blank sample injected immediately prior to II-0, revealed that GE-B4 and B5 were not present in those samples. This ruled out carry over as a possible cause. GE-B4 or GE-B5 were not detected at any time in subject IV. It is interesting to note that subjects I and II had the highest level of intestinal damage (grade 3 of the mucosal damage classified according to the Marsh criteria [33]). Subject III (Marsh grade 2) had only a single point of GE detection and subject (Marsh grade 1) none, suggesting that higher levels of intestinal damage are associated with detection of GEs in blood plasma. Furthermore, GE$\mathrm{A} 4, \mathrm{GE}-\mathrm{A} 5$ and GE-C were not detected in the plasma of any of the subjects at any time. By comparing LC-MS/MS peak areas of the samples with GE standards, in the samples in which it is detected, GE-B4 ranged from 2.2-8.5 nM, while GE-B5 ranged from 2.3-7.9 nM. Thus, in persons with increased intestinal permeability, low nM levels of GE-B4 and GE-B5 can be detected in plasma.

After the "pizza meal," GE-B4 and/or GE-B5 were observed in three out of four subjects. With the exception of sample 90 in subject III, the observation of GE-B4 in a specific sample coincides with the observation of GE-B5. Whether GE-B4 is absorbed as a full intact peptide, or 


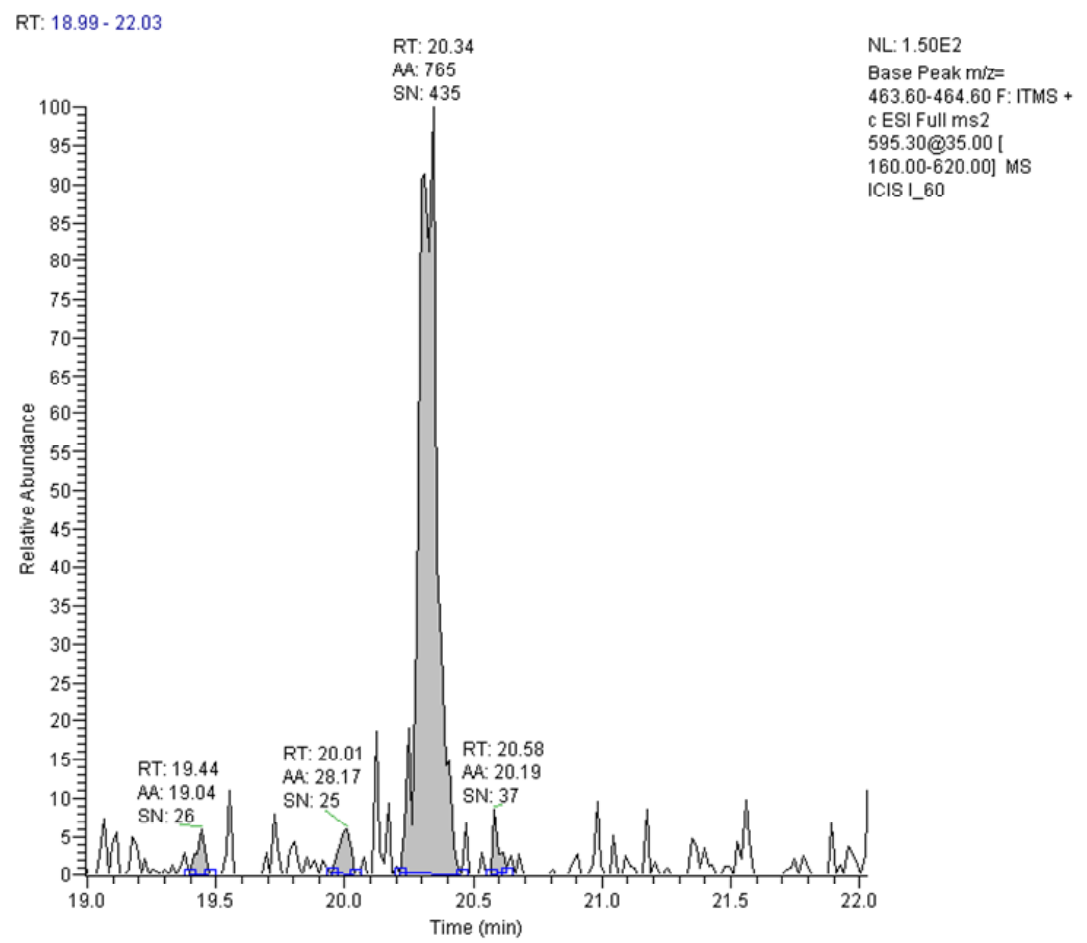

Fig. (5). Extracted ion chromatogram from sample I-60 produced by plotting the ion transition $\mathrm{m} / z$ 595 $\rightarrow \mathrm{m} / z$ 464, with a peak at $16.01 \mathrm{~min}$ that matches the RT of the GE-B5 standard.

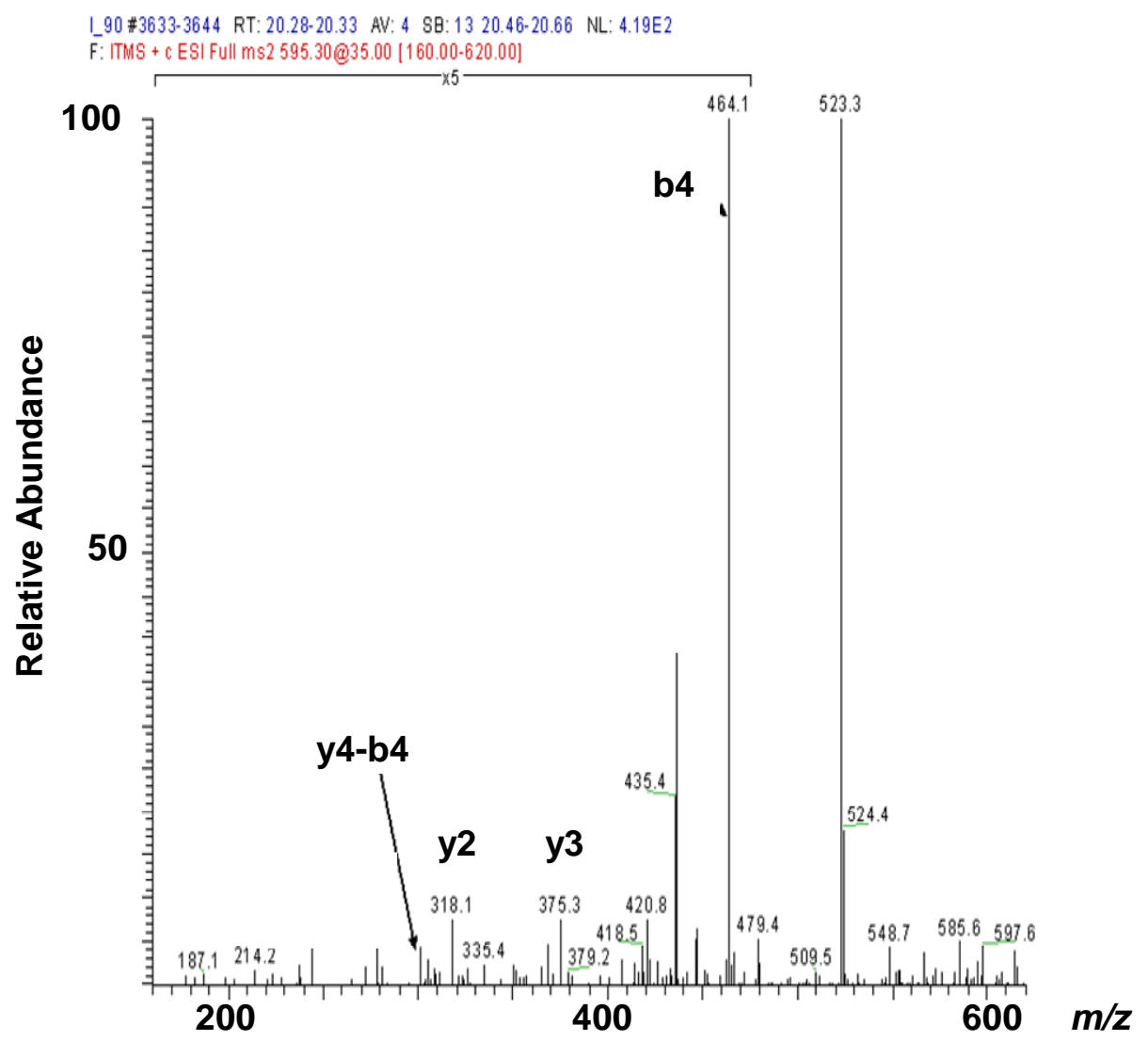

Fig. (6). MS/MS spectrum of $\mathrm{m} / z 595$ from the chromatographic peak at $20.34 \mathrm{~min}$ from sample I-90. 
results from degradation of GE-B5, could not be determined from this study.

The observation of GE-B4 and GE-B5 at time 0 (subject II) is not due to the consumption of the "pizza meal," nor can it be attributed to sample carry over from the instrumentation. Consequently, the detection of these GEs must result from the ingestion of another dietary source of gluten prior to the "pizza meal." It is unclear, however, how much of the GE-B4 and GE-B5 detected in samples II-15 and II-30 is due to the "pizza meal" or due to a source of dietary gluten ingested prior to the experiment.

\section{CONCLUSIONS}

The scope of this study is obviously preliminary and limited to the detection of GE peptides in blood samples obtained from subjects with compromised intestinal lining. This study does not have any quantitative aspects and can not comment on the kinetics of GE production or degradation nor can say if the production and accumulation of GEs in the blood stream occurs under other conditions where the subject's intestinal lining is not compromised. Furthermore, this study cannot comment on any role that GE peptides play, once adsorbed into the blood stream, in the pathology of mental disorders. Only specifically designed studies could clarify if GEs are actually linked to the genesis of mental disorders associated with CD. Lastly, while the data from the four subjects in this study is compelling, a follow up study using a larger sample pool, representative of all degrees of intestinal damage, is necessary before broader conclusions can be made about GE absorption into the blood stream.

\section{ACKNOWLEDGMENTS}

This study was supported by grant 2005 "Identificazione e quantificazione nel liquido cefalo-rachidiano di peptidi oppioidi di origine alimentare" from Fondazione Banco di Sardegna (to Dipartimento-Struttura Clinica MedicaPatologia Speciale Medica, G. Fanciulli) as well as the Mark Diamond Research Fund at the University at Buffalo (to C. L. Pennington). Early support in development of the LCMS/MS method to detect gluten exorphins in biological fluids (to T. D. Wood) was provided from the United Kingdom's Legal Services Commission through AlexanderHarris, Ltd. We would also like to thank Professor Diana Aga and Seamus O'Connor for their assistance and generous use of the centrifuge and freeze dryer.

\section{REFERENCES}

[1] Teschemacher, H. Opioid receptor ligands derived from food proteins. Curr Pharmaceut Design 2003; 9, 1331-44.

[2] Fukudome, S.; Yoshikawa M. Opioid peptides derived from wheat gluten: their isolation and characterization. FEBS Lett 1992; 296, 107-11.

[3] Fukudome, S.; Yoshikawa M. Gluten exorphin C. A novel opioid peptide derived from wheat gluten. FEBS Lett 1993; 316, 17-9.

[4] Fukudome, S.; Jinsmaa, Y.; Matsukawa, T.; Sasaki, R.; Yoshikawa, M. Release of opioid peptides, gluten exorphins by the action of pancreatic elastase. FEBS Lett 1997; 412, 475-79.

[5] Shattock, P.; Whiteley, P. Biochemical aspects in autism spectrum disorders: updating the opioid-excess theory and presenting new opportunities for biomedical intervention. Expert Opin Ther Targets 2002; 6, 175-83.

[6] Hamilton, I.; Cobden, I.; Rothwell, J.; Axon, A.T. Intestinal permeability in coeliac disease: the response to gluten withdrawal and single-dose gluten challenge. Gut 1982; 23, 202-10.
Eonchy, J.G.; Heymans, H.S. Intestinal permeability in pediatric gastroenterology. Scand J Gastroenterol 1992; 194, 19-24.

8] Drago, S.; El Asmar, R.; Di Pierro, M.; Grazia Clemente, M.; Tripathi, A.; Sapone, A.; Thakar, M.; Iacono, G.; Carroccio, A.; D'Agate, C.; Not, T.; Zampini, L.; Catassi, C.; Fasano, A. Gliadin, zonulin and gut permeability: Effects on celiac and non-celiac intestinal mucosa and intestinal cell lines. Scand J Gastroenterol 2006; 41, 408-19.

[9] Pynnonen, P.A.; Isometsa, E.T.; Aronen, E.T.; Verkasalo, M.A.; Savilahti, E.; Aalberg, V.A. Mental disorders in adolescents with celiac disease. Psychosomatics 2004; 45, 325-35.

[10] Zelnik, N.; Pacht, A.; Obeid, R.; Lerner, A. Range of neurologic disorders in patients with celiac disease. Pediatrics 2004; 113, 1672-6.

[11] Eaton, W.W.; Byrne, M.; Ewald, H.; Mors, O.; Chen, C.Y.; Agerbo, E.; Mortensen P.B. Association of schizophrenia and autoimmune diseases: linkage of Danish national registers. Am J Psychiatry 2006; 163, 521-8.

[12] Branski, D.; Troncone, R. Celiac disease: a reappraisal. J Pediatrics $1998 ; 133,181-7$.

[13] Wakefield, A.J.; Puleston, J.M.; Montgomery, S.M.; Anthony, A.; O'Leary, J.J.; Murch, S.H. Review article: the concept of enterocolonic encephalopathy, autism and opioid receptor ligands. Aliment Pharmacol Ther 2002; 16, 663-74.

[14] Fukudome, S.; Shimatsu, A.; Suganuma, H.; Yoshikawa, M. Effect of gluten exorphins A5 and B5 on the postprandial plasma insulin level in conscious rats. Life Sci 1995; 57, 729-34.

[15] Fanciulli, G.; Dettori, A.; Fenude, E.; Demontis, M.P.; Alberico, E.; Delitala, G.; Anania, V. Intravenous administration of the foodderived opioid peptide gluten exorphin B5 stimulates prolactin secretion in rats. Pharmacol Res 2003; 47, 53-8.

[16] Fanciulli, G.; Dettori, A.; Tomasi, P.; Demontis, M.; Gianorso, S.; Anania, V.; Delitala, G. Prolactin and growth hormone response to intracerebroventricular administration of the food opioid peptide gluten exorphin B5 in rats. Life Sci 2002; 71, 2383-90.

[17] Fanciulli, G.; Dettori, A.; Demontis, M.P.; Anania, V.; Delitala, G. Serum prolactin levels after administration of the alimentary opioid peptide gluten exorphin B4 in male rats. Nutr Neurosci 2004; 7, 535.

[18] Ahern, J.A.; Gatcomb, P.M.; Held, N.A.; Petit, Jr., W.A.; Tamborlane, W.V. Exaggerated hyperglycemia after a pizza meal in wellcontrolled diabetes. Diabetes Care 1993; 16, 578-80.

[19] Capaldo, B.; Gastaldelli, A.; Antoniello, S.; Auletta, M.; Pardo, F.; Ciociaro, D.; Guida, R.; Ferrannini, E.; Sacca, L. Splanchnic and leg substrate exchange after ingestion of a natural mixed meal in humans. Diabetes 1999; 48, 958-66.

[20] Nappo, F.; Esposito, K.; Cioffi, M.; Giugliano, G.; Molinari, A.M.; Paolisso, G.; Marfella, R.; Giugliano, D. Postprandial endothelial activation in healthy subjects and in type 2 diabetic patients: role of fat and carbohydrate meals. J Am Coll Cardiol 2002; 39, 1145-50.

[21] Baussant, T.; Bougueleret, L.; Johnson, A.; Rogers, J.; Menin, L.; Hall, M.; Aberg, P.-M.; Rose K. Effective depletion of albumin using a new peptide-based affinity medium. Proteomics $2005 ; 5,973-$ 77 .

[22] Gambus, P.L.; Schnider, T.W.; Minto, C.F.; Youngs, E.J.; Billard, V.; Brose, W.G.; Hochhaus, G.; Shafer, S.L. Pharmacokinetics of intravenous dynorphin $\mathrm{A}(1-13)$ in opioid-naive and opioid-treated human volunteers. Clin Pharmacol Ther 1998; 64, 27-38.

[23] Hussain, M.A.; Rowe, S.M.; Shenvi, A.B.; Aungst, B.J. Inhibition of leucine enkephalin metabolism in rat blood, plasma and tissues in vitro by an aminoboronic acid derivative. Drug Metab Dispos $1990 ; 18,288-91$.

[24] Mosnaim, A.D.; Wolf, M.E.; Nguyen, T.D.; Puente, J.; Freitag, F.; Diamond, S. Degradation kinetics of leucine5-enkephalin by plasma samples from healthy controls and various patient populations: in vitro drug effects. Am J Ther 2000; 7, 185-94.

[25] Rai, A.J.; Gelfand, C.A.; Haywood, B.C.; Warunek, D.J.; Yi, J.; Schuchard, M.D.; Mehigh, R.J.; Cockrill, S.L.; Scott, G.B.; Tammen, H.; Schultz-Knappe, P.; Speicher, D.W.; Vitzthum, F.; Haab, B.B.; Siest, G.; Chan, D.W. HUPO Plasma Proteome Project specimen collection and handling: towards the standardization of parameters for plasma proteome samples. Proteomics 2005; 5, 3262-77.

[26] Yuan, X.; Desiderio, D.M. Human cerebrospinal fluid peptidomics. J Mass Spectrom 2005; 40, 176-181. 
[27] Harper, R.G.; Workman, S.R.; Schuetzner, S.; Timperman, A.T.; Sutton, J.N. Low-molecular-weight human serum proteome using ultrafiltration, isoelectric focusing, and mass spectrometry. Electrophoresis 2004; 25, 1299-1306.

[28] Fanciulli, G.; Azara, E.; Wood, T.D.; Dettori, A.; Delitala, G.; Marchetti, M. Quantification of Gluten Exorphin A5 in cerebrospinal fluid by liquid chromatography-mass spectrometry. J Chromatogr $B$ 2006; 833, 204-9.

[29] Feighery, C. Fortnightly review: coeliac disease. BMJ 1999; 319: 236-9.

[30] Makarov, A. Electrostatic Axially Harmonic Orbital Trapping: A High-Performance Technique of Mass Analysis. Anal Chem 2000; $72,1156-62$.
[31] Hardman, M.; Makarov, A.A. Interfacing the Orbitrap Mass Analyzer to an Electrospray Ion Source. Anal Chem 2003; 75, 16991705.

[32] Makarov, A.; Denisov, E.; Kholomeev, A.; Balschun, W.; Lange O.; Strupat, K.; Horning, S. Performance Evaluation of a Hybrid Linear Ion Trap/Orbitrap Mass Spectrometer. Anal Chem 2006; 78, 2113-20.

[33] Marsh, M.N. Gluten, major histocompatibility complex, and the small intestine. A molecular and immunobiologic approach to the spectrum of gluten sensitivity ('celiac sprue'). Gastroenterology $1992 ; 102,330-54$ 